\title{
Forma socio-organizacional del vínculo a priori entre investigación y enseñanza de postgrado*
}

\author{
Fuenmayor, Ramsés**
}

\section{Resumen}

¿Cuál es la condición de posibilidad del vínculo entre la investigación universitaria y la enseñanza de postgrado? Ante esta interrogante se escribe este artículo. Como resultado de un examen crítico de las bases lógicas de pregunta misma, ésta se transforma en “¿Cuál forma organizacional permite un vínculo a priori entre investigación y enseñanza?" La respuesta se concentra en la idea de "práctica" elaborada por Alasdair Maclntyre. Se argumenta cómo esa particular forma socio-laboral llamada "práctica", unida a una cierta concepción de universidad, constituye la tierra socio-organizacional que hace posible una auténtica vinculación entre investigación y enseñanza de postgrado. Fi nalmente, con fines ilustrativos, se resume el modo particular como se hizo posible la vinculación entre la investigación y la enseñanza de postgrado en el caso de la Sistemología Interpretativa.

Palabras clave: Sistemología interpretativa, Investigación, enseñanza, universidad, formas socio-organizacionales.

\section{An A Priori Form of Social-Organizational Linking Between Research and Teaching}

\section{Abstract}

What is the true possibility of a relationship between university research and post-graduate teaching? In answer to this question, this article is written. As a result of a critical analysis of the logical basis of the questions itself, it can be transformed into "Which organizational form would permit an a priori relationship between research and teaching? The answer is found in the idea of "practice" elaborated by Alasdair MacIntyre. He argues that this particular social-labor form called "practice", to-

Recibido: 02-09-19 . Aceptado: 02-05-03

* Este ensayo forma parte del texto de una conferencia dictada en las "I Jornada de Investigación y Postgrado" de la Universidad del Zulia. Maracaibo, Junio 2002.

** Centro de Investigaciones en Sistemología Interpretativa. Facultad de Ingeniería. Universidad de Los Andes. 
gether with a certain conception of university, constitutes the social-organization sub-soil that makes possible an authentic relationship between research and post-graduate teaching. Finally, for illustrative purposes, the particular mode that makes possible this relationship between research and postgraduate teaching is explained in the case of interpretive systems science.

Key words: Interpretive systems science, research, teaching, university, social-organizational forms.

\section{La condición a priori del vínculo entre investigación y enseñanza de postgrado}

Cuando la "vinculación entre investigación y enseñanza de postgrado” se propone como tema de discusión, con frecuencia se piensa el asunto del siguiente modo: “¿Cómo crear una vinculación a posteriori entre dos actividades -investigación y enseñanza de postgrado- que existen de manera aislada a priori?". Algunas veces, aquellos que así se plantean el problema van más allá de la mera inquietud instrumental y aceptan que antes de responder la pregunta organizacional sobre cómo crear tal vinculación es necesario plantearse la pregunta por los posibles y deseables modos de vinculación. Pero aún dando este salto de lo meramente instrumental a lo práctico, generalmente se parte del supuesto inconsciente de que hay una sola y universal estructura lógica dentro de la cual se puede pensar este problema. Tal estructura se puede plantear del siguiente modo: Premisa básica: $\mathrm{X}$ existe como tal sin necesidad de $Y$ y viceversa (es decir, $\mathrm{Y}$ y $\mathrm{X}$ son ontológicamente independientes). Problema: ¿Cómo crear un vinculación entre $Y$ y $X$ ? (A veces, decía, se considera que esta pregunta debe estar precedida por la consideración de los posibles y deseables modos de vinculación).
Como se puede ver, la estructura lógica del problema está fundada en una suposición ontológica; a saber, que el ser de cada cosa es independiente de las vinculaciones que se puedan establecer con otras cosas. Se trata de una estructura ontológica dominante (desde que el atomismo ontológico comenzó a dominar la ciencia clásica) que predetermina la estructura lógica del problema y, clara está, sus posibles soluciones.

Quisiera comenzar por cuestionar el hecho de que ésta sea la única posible estructura lógica que comande de manera determinante la formulación del problema. Aunque resulte extraño ante el modo dominante de pensar el asunto de la vinculación entre $\mathrm{Y}$ y $\mathrm{X}$, es posible pensar que la condición ser de $X$ y de $Y$ dependan de la relación entre $Y y X$. Concebido así, Y y X no tienen primacía ontológica con respecto a su relación. Claro está, con esto no se quiere decir que tal relación sea posible $\sin \mathrm{X}$ y $\sin \mathrm{Y}$. En este caso, la pregunta por la vinculación entre $\mathrm{Y}$ y X es una pregunta por el ser de $\mathrm{X}$ y de $Y$ en su ontológica vinculación.

Las consecuencias de plantearse en serio este otro modo ontológico son tremendas en relación con muchos aspectos de nuestra vida humana (piénsese por ejemplo la relación entre los seres humanos); sin embargo, debemos aquí abandonar el asunto ontológico general 
para ocuparnos del problema específico de la vinculación entre investigación yenseñanza de postgrado. En este campo particular, quisiera proponer la idea de que tal vinculación puede entenderse más a fondo y con mejores consecuencias prácticas bajo la segunda modalidad ontológica; a saber: que la condición de ser de la investigación y de la enseñanza de postgrado dependen de su vinculación. Puesto en otras palabras, quisiera proponer que el asunto de la vinculación entre estas dos actividades no debe ser pensado como posterior a la existencia de tales actividades. ¿Cuál es, entonces, esta vinculación entre enseñanza de postgrado e investigación que le brinda su condición de ser a sendas actividades? ¿Cómo pensar la investigación y cómo pensar la enseñanza de postgrado si partimos del supuesto de que la condición de ser de ambas depende de su vinculación?
Comencemos por ofrecer una primera y sucinta respuesta ante esta última pregunta:

Investigación: Si en su forma más básica se entiende por investigación la búsqueda de conocimiento, de propósito y sistemática, con pretensión de verdad; y si aceptamos que todo conocimiento y su búsqueda es un hecho social; entonces deberíamos aceptar que la enseñanza del conocimiento está allí como motivo y como consecuencia del acto de investigación. Explico:

La búsqueda de conocimiento se da sobre la base del lenguaje básico y sobre la de ciertos "juegos lingüísticos" propios del área cognoscitiva en cuestión. Esto quiere decir que la actividad de investigación, por muy solitaria que ésta sea, es una jugada de un "juego lingüístico" y que, como tal, necesariamente parte del tejido social y busca desembocar en el tejido social. La desembocadura de

Estoy usando esta frase en el sentido que, según entiendo, le da Ludwig Wittgenstein en su libro "Investigaciones Filosóficas" (1978). Uno de los ejemplos que Wittgenstein usa para caracterizar lo que llama "juego lingüístico" son esos juegos infantiles en los que los niños cantan una canción mientras realizan, en conjunto, una serie de actividades ("acciones dentro de las cuales se teje un lenguaje" p. 5) relacionadas con la letra de la canción (e.g. "A la víbora de la mar"). En general, pienso que se trata de un modo de hablar "entretejido" con un modo de actuar (o, de un modo de actuar entretejido con un modo de hablar), diferente de otros modos de "hablar-actuar", en el que el modo de hablar le da sentido al modo de actuar (además, lo comanda, comenta, reporta, proyecta); $y$ en el que el modo de actuar realiza, le da sentido, concreción y ubicación al modo de hablar; de manera que no es posible ni pensar ni vivir el modo de actuar sin el modo de hablar, ni el modo de hablar sin el modo de actuar. La unidad indisoluble que ellos constituyen se llama "juego lingüístico". En un "juego lingüístico", las palabras son comprensibles en términos del contexto brindado por todo el "juego lingüístico". Y este "ser comprensible" no significa que ellas "signifiquen" algo en el juego lingüístico (como una palabra que buscamos en un diccionario "significa" su definición), sino que tienen un (o unos) "uso(s)" en el "juego lingüístico". Lo que sigue a continuación hace uso de la noción de "juego lingüístico" y, en general, de la investigación sobre el lenguaje que Wittgenstein emprende en sus "Investigaciones Filosóficas". 
la actividad de investigación en el tejido social es la enseñanza. No puede pues pensarse en el acto de investigación sin la enseñanza como causa teleológica del mismo.

Enseñanza: Permítaseme por ahora referirme a la enseñanza universitaria en general. Más tarde mostraré que si mis argumentos son válidos para la enseñanza universitaria en general mucho más lo son para la enseñanza universitaria de postgrado en particular.

Desde el punto de mira del aprendiz, la enseñanza tiene como propósito fundamental aprender a aprender. Los conocimientos particulares que se imparten en el proceso de enseñanza son sólo medios para que el individuo aprenda a aprender en un cierto campo del conocimiento. Aprender a aprender es aprender a investigar. Así, por ejemplo, enseñar bien la práctica médica requiere enseñar el arte de investigar en medicina: desde esa investigación constitutiva del meollo de la práctica médica que es el proceso de diagnóstico de una enfermedad en un paciente particular, hasta la investigación destinada a encontrar la cura de enfermedades en general.

Tal como antes anuncié, lo anterior es una muy sucinta y esquemática respuesta ante la pregunta "¿Cómo pensar la investigación y cómo pensar la enseñanza de postgrado si partimos del supuesto de que la condición de ser de ambas depende de su vinculación?". Como tal, es sólo una posible indicación abs- tracta del punto básico de contacto entre la investigación y la enseñanza. Sin embargo, dice muy poco sobre la forma social en la que se puede insertar la vinculación a priorientre esas actividades humanas que llamamos "investigación" y "enseñanza". Lo que quiero decir es que esa respuesta deja de lado un hecho fundamental: la investigación y la enseñanza son actividades humanas cuyo ser radica en una forma de convivencia humana de carácter cotidiano. Esta forma de convivencia le brinda su sentido social a la actividad humana en cada caso concreto. Por esta razón, bajo un interés práctico, la pregunta por la posibilidad de la vinculación entre investigación y enseñanza no puede evadir la cuestión sobre la forma social de convivencia humana donde se asientan estas actividades y su vínculo. En efecto, como espero mostrar a continuación, hay una tal forma de convivencia en donde es posible comprender y cultivar la vinculación a priorientre investigación y enseñanza de postgrado. Es más, hasta dónde puedo ver, es la única forma de convivencia en la que tal vinculación puede tener un auténtico sentido. Me refiero a las prácticas, en el sentido que Alasdair Maclntyre le da al término en su libro: "After Virtue". Veamos:

\section{Práctica como forma organizacional y enseñanza}

Según Maclntyre $(1985)^{2}$, [Una práctica es] una forma coherente y compleja de actividad humana cooperativa, 
socialmente establecida, mediante la cual se realizan bienes internos a esa forma de actividad, en la medida en que se intentan alcanzar esos patrones de excelencia que son apropiados para -y que parcialmente definen a- esa forma de actividad; con el resultado de que los poderes humanos para alcanzar excelencia y las concepciones de los fines y bienes envueltos son sistemáticamente mejorados.

(Piénsese en una buena escuela de música).

Una virtud es una cualidad humana adquirida, cuya posesión y ejercicio tiende a permitirnos el logro de los bienes internos de una cierta práctica; y cuya fatta nos impide efectivamente alcanzar dichos bienes (Maclntyre, 1985).

(Piénsese en la idea de virtud implícita en la expresión "es un virtuoso del violín").

Vale la pena extraer ciertas consecuencias de esas dos definiciones que serán importantes para pensar el asunto de la vinculación entre la investigación y la enseñanza de postgrado en el ambiente universitario. Para ello, es importante insistir en que una práctica es una forma coherente y compleja de actividad humana cooperativa y socialmente establecida, la cual cumple las siguientes condiciones:

1. Mediante esa actividad se logran ciertos bienes; es decir, ciertos productos tangibles o intangibles de la actividad que se consideran como buenos.

2. La calidad (la condición de "buenos") de estos productos es siempre mejorable.

3. La actividad propia de la práctica está esencialmente acompañada por una constante voluntad de lograr la excelencia del bien.

4. Tal voluntad de lograr la excelencia del bien se traduce en una voluntad de "hacer bien el bien". Es decir, que la condición de "excelencia" se refiere tanto al producto de la actividad como a la actividad que lo produce.

5. En otros términos, podría decirse que el bien que persigue una práctica convoca la actividad humana para su creación. Bajo esta perspectiva, ese bien es creador y recreador de actividad humana, pues convoca la reunión de seres humanos en torno de sí. De esta manera, el bien de una práctica mancomuna. Pero recordemos que la condición de bien se va definiendo por lo bien que se realice la actividad humana mancomunada que lo procura. Por tanto, el bien va definiendo el modo de mancomunidad que lo hace posible. La práctica es, pues, esa actividad humana creadora y recreadora de un bien que recrea lo humano en su forma mancomunitaria.

6. Las virtudes propias de una práctica son esas cualidades humanas necesarias para crear, recrear y apreciar el bien a lo largo del camino de la búsqueda de su excelencia. Nótese que las virtudes no sólo permiten producir el bien de esa práctica en su mejor condición posible, sino que permiten apreciarlo como tal. En otras palabras, aquél que no posea las virtudes propias de esa práctica no podrá apreciar cabalmente el grado de excelencia del bien producido por dicha práctica. Esta apreciación interna del bien será llamada "cara interna del bien". 
7. Sin embargo, para que la prácticasea socialmente establecida, el bien (o bienes) que ella produce deberá poseer también una cara externa; esto es, una cara que permita darle sentido social a la práctica en cuestión.

Lo anterior implica que: primero, la vida de una práctica se sustenta en esa voluntad colectiva de mejorar el bien que le da la razón de ser a dicha práctica. Es decir, la vitalidadde una prácticase funda en el continuo ejercicio y mejoramiento de las virtudes que son propias de dicha práctica -virtudes que, recuérdese, son necesarias, no sólo para producir el bien en su estado de mayor excelencia posible, sino que, al mismo tiempo, son necesarias para apreciar la excelencia de ese bien. Sustráigase la fuerza de aquella voluntad, o, sustráigase el ejercicio y mejoramiento de las virtudes, y se tendrá un cadáver de práctica. Obsérvese que esta condición de vida de una práctica es propia de toda práctica, independientemente de cuál sea el bien que produce -así como decimos que la condición de vida de cualquier mamífero depende de ciertas características funcionales biológicas básicas (por ejemplo, de que su corazón funcione adecuadamente), independientemente de cuál sea el mamífero del caso.

Segundo, la ausencia de virtudes en una práctica se da en un rango que va desde la mediocridad hasta el vicio. La mediocridad es la simple y pasiva ausencia de virtud. La mediocridad es dañina para la práctica por ser mera ausencia de virtud, falta de vida. El vicio es una cualidad cuyo ejercicio daña activamente a la práctica y a su bien.

Tercero, puesto que las virtudes son necesarias para producir y para apre- ciar el bien de una práctica, las personas más virtuosas son las llamadas a guiar y coordinar las actividades de la práctica. Esta guía y coordinación es, al mismo tiempo, un proceso de enseñanza continua de las virtudes propias de la práctica en cuestión.

Alcanzamos así el punto de enlace entre la explicación sobre la noción de práctica que hemos venido desarrollando y el tema central de este opúsculo sobre la vinculación entre investigación y enseñanza. Una práctica (en buen estado) no puede prescindir de un proceso continuo de enseñanza de las virtudes requeridas para producir su bien de manera excelente. No se trata de que a las actividades propias de la práctica se le añadan actividades de enseñanza. No, la enseñanza es un aspecto vital constitutivo de todo lo que se hace en la práctica. Una práctica a la que haya que agregarle la actividad de enseñanza es disfraz de práctica 0 , al menos, una práctica en mal estado. Es por ello que afirmé al comienzo de esta explicación sobre las prácticas que el nicho social necesario para una auténtica vinculación a priori entre investigación y enseñanza de postgrado es, precisamente, el de una práctica.

Lo que quiero decir -y permítaseme insistir en el punto- es que la enseñanza es la expresión de la recreación de lo humano en su forma mancomunitaria en torno a un bien. La enseñanza es la consecuencia necesaria de aquella voluntad de lograr la excelencia a la que antes nos referíamos. Pero -y aquí alcanzamos un punto crucial en nuestro discurso- si la enseñanza es una actividad consustancial de cualquier buena práctica, ¿cuál es entonces ese bien que se pretende alcan- 
zar con la práctica universitaria para que exista una auténtica vinculación entre investigación y enseñanza? La respuesta, si se ha entendido bien la noción de práctica, es perogrullesca: -ese "bien" es el que se busca con la investigación. Por esta razón considero que sólo si entendemos la universidad como una práctica de investigación podremos tener una auténtica enseñanza de postgrado.

Sin embargo, el asunto no es tan simple, porque, en realidad, lo que estamos preguntando es por una concepción de universidad en la cual pueda lograrse una auténtica vinculación a priori entre investigación y enseñanza de postgrado. Por esta razón, debo dedicar unos minutos a un breve enunciado de los que considero principios rectores ${ }^{3}$ de una concepción de universidad en la cual se logre una síntesis armoniosa entre investigación y enseñanza; en particular, entre investigación y enseñanza de postgrado. Esto no es otra cosa -ya enfilando el discurso hacia el asunto de la vinculación concreta entre la investigación y la enseñanza de postgrado en sistemología interpretativa- que un breve enunciado de la concepción de universidad que ha movido nuestra práctica de sistemología interpretativa.

\section{El bien que persigue la práctica universitaria}

La misión (o papel social) de la universidad no puede confundirse con la de otras instituciones de la sociedad: tiene que ser específica, propia y única de la universidad. Por otra parte, esa misión debe presentar una unidad a la cual concurren las diferentes actividades o partes de la institución. Es esta especificidad institucional y esta unidad sistémica las que brindan su ser a la universidad.

Pienso que la misión general de la universidad, entendida ésta como una institución propia de lo más esencial de la cultura Occidental, es la siguiente:

La universidad es la casa del cultivo de la verdad ${ }^{4}$. La verdad es la apertura donde aquello que es el caso se muestra en su proteico ser a partir de su fundamento -de lo que lo hace posible. El cultivo de la verdad se realiza mediante el cultivo del saber (con pretensión de verdad) $y$ de las artes -en general, de las formas excelsas de la cultura.

"Cultivo del saber" significa creación y difusión del saber, ambas dentro de una misma práctica. Según Maclntyre (1985), el cultivo del saber entendido como práctica humana une en un sólo

3 Parte del enunciado de tales principios, así como parte de la exposición anterior referente al tema de las prácticas, ha sido tomado de mi artículo: "Entre la mediocridad institucional y el dominio imperial de lo instrumental" (2001b).

4 Para una discusión profunda sobre el asunto de la verdad en el sentido aquí usado véase Hejdegger (1993). 
concepto la noción de investigación y enseñanza. No tiene sentido, en la universidad, concebir una actividad de enseñanza desligada de la investigación, ni una investigación destinada a ocultar el producto de su esfuerzo. Por esta razón, la actividad básica universitaria es la investigación, dentro de una organización que permita, por una parte, la formación de los que continuarán la labor de investigación, y, por la otra, la continua difusión de ese saber en la sociedad nacional e internacional. Pero, aquí es necesario aclarar algo en relación con el uso que hago de la palabra "investigación": Cuando hablo de "investigación" no me refiero simplemente a esa actividad productora de conocimientos, tarde o temprano vinculados con lo tecnológico, útiles para el aparato industrial y evaluados en relación con su papel en el mercado. No me refiero a la búsqueda de conocimientos, entendidos éstos como meros instrumentos tecnológicos. La investigación es la búsqueda incesante de la verdad. Es cierto: es la búsqueda de conocimientos, pero de conocimientos que de inmediato son sometidos a una crítica que no acepta confinarse ni en los límites de lo instrumental ni en los de una disciplina particular; conocimientos que son pasados por el más riguroso cedazo de preguntas que van más allá del criterio de "certeza". Preguntas tales como: "¿Sobre cuáles formas ontológicas, epistemológicas, políticas y morales se sustentan estos conocimientos? ¿Al servicio de qué y de quienes serán usados? ¿Por qué? y ¿para qué?" son consustanciales a la vida universitaria.

\section{La enseñanza de pregrado y la postgrado}

A partir de los anteriores principios -que apretadamente resumen una concepción de universidad- no es difícil percatarse de la diferencia entre la enseñanza de pregrado y la de postgrado en relación con la vinculación entre éstas y la investigación:

En general, la práctica del cultivo de la verdad en Occidente se ha encausado en tradiciones que comenzaron en la antigua Grecia pre-socrática. En particular, la búsqueda de conocimientos en disciplinas específicas también se encausa en tradiciones -algunas longevas, otras de reciente data- en las cuales se van sedimentando ciertas habilidades y conocimientos básicos sobre los cuales, a su vez, se construyen y destruyen otros más recientes. En todo caso, el proceso de investigación en una cierta disciplina podría compararse con la construcción de un edificio en la que, a lo largo de la historia, se avanza desde abajo hacia arriba. En la parte superior se realiza la mayor parte de la actividad constructora de nuevos pisos fundada en pisos anteriores. Sin embargo, ocasionalmente, esta misma actividad revela la necesidad de derrumbar pisos antes construidos para reiniciar la edificación a partir de este nivel. Lo que quiero decir con esta burda metáfora es que la actividad de investigación se mueve sobre la base de lo ya investigado en la tradición en cuestión, cuestionando y sedimentando (gracias al cuestionamiento) esa misma base. Por 
esta razón la enseñanza propia de esta práctica se organiza en varias etapas: Las iniciales o básicas, en contacto menos estrecho con la avanzada de la investigación, se deberían encargar de la enseñanza de esas habilidades y conocimientos sedimentados a lo largo de la historia. Las más avanzadas se van acercando al calor de la "investigación de punta" -la punta que abre camino-. Es por ello que la vinculación entre la enseñanza de postgrado y la investigación de avanzada es mucho más activa y obvia. Pero, ¿cómo es esa vinculación?

El modo de vinculación varía según la disciplina, pero creo que, en general, el lugar común de una buena vinculación entre enseñanza de postgrado e investigación lo aporta, de manera muy simple, la idea de práctica: los aprendices se insertan en el proceso de investigación y aprenden lo que se requiera para poder contribuir con éste. Me atrevo a decir que un programa de postgrado que, grosso modo, no funcione de esta manera en relación con la investigación -que por ejemplo esté constituido por meras clases magistrales donde se imparten conocimientos básicos- no merece llamarse programa de postgrado.

Me resta ahora, para terminar de cumplir con la pregunta que se me ha formulado, referir muy brevemente qué es lo específico en la sistemología interpretati- va en relación con esa vinculación entre investigación y enseñanza de postgrado.

\section{La investigación y la enseñanza de postgrado en la sistemología interpretativa: Sus inicios}

La Sistemología Interpretativa nació, hace ya un poco más de 20 años, de la reunión de dos interrogantes, de dos inquietudes académicas: La primera interrogante surgió cuando los que iniciamos esta disciplina, que ahora llamamos Sistemología Interpretativa, éramos jóvenes profesores de la Escuela de Ingeniería de Sistemas de la Universidad de Los Andes. La Ingeniería de Sistemas era, para ese entonces, una no muy bien definida disciplina y carrera universitaria cuyo propósito parecía ser -así lo entendíamos a partir de nuestras lecturas- el del estudio de situaciones problemáticas complejas ${ }^{5}$ bajo el enfoque de sistemas con una orientación práctica ${ }^{6}$. El enfoque de sistemas, directriz de la joven disciplina, se ofrecía como la intención de estudiar y actuar bajo la premisa "holística" de que el todo trasciende la reunión de las partes. Dicha intención estaba fundada en un ataque al marcado énfasis reduccionista-analítico de la práctica científica y tecnológica. Así, el enfoque de sistemas atacaba el modo reduccionista y analítico

5 Las organizaciones de actividades humanas fueron el campo principal de estudio de tales "situaciones problemáticas complejas".

6 Cuando decimos que tales estudios tenían una "orientación práctica" queremos decir que pretendían derivar racionalmente cursos de acción a partir del estudio en cuestión. 
del proceder usual científico en los siguientes términos: "aislar un fenómeno de su contexto (reduccionismo) y comenzar su estudio por una separación en sus partes implica perder de vista la condición holística de cualquier fenómeno". En esa época ya comenzaban a proliferar métodos sistémicos para el estudio de organizaciones de actividades humanas -objeto predilecto de la nueva disciplina-. Sin embargo, asombrosamente, la pregunta básica ante el lema fundamental del enfoque de sistemas quedaba desatendida por los estudiosos del "movimiento de sistemas".

En efecto, preguntas tales como: ¿cómo es posible que el todo trascienda la reunión de sus partes? ¿cómo es posible el conocimiento del todo en cada caso? ¿por qué la práctica científica parece ignorar esa intuición holística básica? eran continuamente evadidas por los precursores y seguidores de la nueva disciplina. Esas preguntas, obviamente, exigían la formulación de una base teórica para el enfoque de sistemas. Esa base teórica no sería otra cosa que una ontoepistemología que diera cuenta de la condición holística de lo que ocurre y de la posibilidad de su estudio ${ }^{7}$.

Creímos, aquellos jóvenes profesores de la Escuela de Ingeniería de Sistemas, que contribuir con el desarrollo de tal teoría sería una digna tarea dentro de nuestro contexto académico. La impor- tancia de tal tarea se hacía más acuciante al constatar que la práctica de la ingeniería y ciencia de sistemas, en nuestra Escuela de Ingeniería de Sistemas y en otras instancias universitarias del mundo, parecía alejarse de aquellos principios que considerábamos originales. En efecto, la práctica de la ingeniería de sistemas parecía ser más un poderoso instrumento para diseñar y mantener complejas organizaciones de actividades humanas (de las que jamás se pregunta por su sentido o papel social), que una práctica destinada a comprender y cuestionar el sentido de tales organizaciones. Creímos que este marcado instrumentalismo iba de la mano con el descuido que la joven disciplina mostraba por comprender su propio principio sistémico.

Más adelante, gracias a lo andado en ese camino de investigación, fuimos comprendiendo que el instrumentalismo, más allá de ser una tendencia, un interés, que se adueñaba de ciertas disciplinas (como paradójicamente ocurría con la ingeniería de sistemas), era un modo de ser cultural que estaba amenazando gravemente la posibilidad de hacer sentido holístico de lo que ocurre. Quiero decir que observamos un deterioro de la posibilidad del sentido holístico no sólo en la ciencia y en la tecnología sino en la vida cotidiana de aquellos que vivimos en culturas occidentales y, muy especialmente, de los que vivimos en culturas "occidenta- 
lizadas"8. Esta observación hizo saltar nuestro interés por la promoción del estudio del sentido holístico de organizaciones de actividades humanas a un interés mucho más general por la recuperación del sentido holístico de la vida cotidiana.

En relación con el tema general que tratamos en este artículo quisiera abrir un paréntesis para llamar la atención sobre lo siguiente: Esta primera inquietud de la sistemología interpretativa presentaba dos caras: una inquisitiva y una política. La cara inquisitiva es obvia: queríamos comprender cómo es posible que el todo trascienda a la reunión de las partes y cuáles consecuencias epistemológicas se derivan de esta condición ontológica. Pero, del otro lado de esta cara inquisitiva, menos visible, palpitaba una cara política: estábamos, por una parte, intentando reformar una disciplina -la de sistemas- que intuíamos mal fundamentada y minada por una contradicción fundamental entre su principio holístico y su quehacer instrumental. Pero, por otra parte, habitaba en nosotros el sueño de contribuir con un mundo mejor en el que lo que ocurre pueda tener un sentido más rico y pleno. Digo que era una cara política porque de eso trata la política en su forma original y auténtica: de la participación pública de los seres humanos destinada a mejorar sus sociedades. Espero que el lector note cómo, en este caso, ambas caras, la política y la inquisitiva, son "caras de una misma moneda"; una no podría ser sin la otra. Pero, además, es importante notar que el aspecto político implicaba un enfrentamiento contra lo que lucía como dominante: el dominio de la racionalidad instrumental en la ingeniería de sistemas, en la ciencia y en la tecnología en general, y, más allá, en la vida de las sociedades modernas. Todo esto implicaba un compromiso intelectual y afectivo con nuestra actividad laboral que iba mucho más allá de la mera rutina.

La segunda interrogante alude al objeto de estudio privilegiado de la nueva disciplina de sistemas eran las organizaciones de actividades humanas (organizaciones tales como las empresas, las instituciones públicas, las organizaciones de acción comunitaria, las cooperativas, entre otras). En este campo de las organizaciones de actividades humanas, nos llamaba la atención un fenómeno que difícilmente escapaba a la vista de alguien que con detenimiento y actitud reflexiva observara y pensara en las organizaciones públicas venezolanas; a saber: la gran brecha que existía entre el discurso justificador de estas instituciones y su actual papel social. A esta cualidad general que creíamos encontrar en muchas de las instituciones venezolanas la llamamos "esquizofrenia institucional".

Llamo culturas "occidentalizadas" a las que devinieron de la imposición de una cierta forma, generalmente marginal, de la cultura occidental a otra cultura que originalmente era no-occidental. Las instituciones de estas culturas occidentalizadas guardan la apariencia de ser occidentales, aunque en su interior alberguen importantes contradicciones culturales. Claros ejemplos de culturas occidentalizadas son las sociedades latinoamericanas y buena parte de las sociedades "subdesarrolladas". 


\section{¿Ocurría realmente este fenómeno} de "esquizofrenia institucional" en muchas de las instituciones públicas venezolanas? De ser así, ¿Cómo se caracterizaba en los casos particulares? ¿Cómo se mantenía? ¿Por qué? ¿A quién servía y cómo? ¿Cómo se vinculaba con nuestro supuesto intento de modernización como país, como sociedad? ¿Qué papel jugaba la historia del encuentro de nuestras culturas constitutivas en este asunto? -esas y otras eran preguntas que salían al paso del inicio de nuestro andar académico ${ }^{9}$.

Esta segunda inquietud académica también estaba, al igual que la primera, acompañada por un interés político (en el sentido original de la palabra): considerábamos que tendríamos mejores organizaciones en la medida en que estuviésemos en mayor capacidad de discutir sus sentidos actuales y deseables.

Las dos inquietudes -la fundamentación del enfoque de sistemas y la comprensión del fenómeno de esquizofrenia institucional- se encontraron fácilmente en la formulación de un proyecto de vida académica: Desarrollar una teoría de sistemas a partir de la cual fuera posible estudiar el sentido socio-cultural de las instituciones y prácticas sociales de nuestro medio. Pero, ¿qué pertinencia tiene todo este cuento en relación con el modo como en la sistemología interpretativa se dio el vínculo entre investigación y enseñanza de postgrado?

\subsection{Las condiciones específicas de la sistemología interpretativa que contribuyeron a crear una práctica universitaria sostenida en un vínculo a priori entre investigación y enseñanza de postgrado}

Creo que hubo tres condiciones fundamentales, poco comunes en las universidades de países occidentalizados, que contribuyeron de manera decisiva en el modo de vinculación a priori que se observa entre investigación y enseñanza de postgrado en la práctica de la sistemología interpretativa. Las dos primeras de estas condiciones están a la vista en la anterior narración sobre el origen de nuestra disciplina:

1) Nuestra unidad académica no surgió de un acto de "implante institucional", sino de una problemática inquisitiva que fluyó por un cauce institucional. La mayor parte de las unidades académicas (departamentos, centros, institutos) de nuestras universidades surgen de la transferencia o "implante" de un cuerpo de conocimientos (el cual generalmente ya cuenta con unidades académicas similares en países desarrollados) ya iniciado en otros lugares. Este modo de "implante" no necesariamente permite que el

9 Esta segunda inquietud dio lugar a una teoría sistémico-interpretativa de organizaciones (Fuenmayor, 2001a), basada en la onto-epistemología sistémico interpretativa, y a una serie de estudios de organizaciones específicas. En la página web del Centro de Investigaciones en Sistemología Interpretativa (http://www.ing.ula.ve/ sisint/) puede verse un panorama más amplio de nuestra actividad académica. 
espíritu inquisitivo derivado de una cierta problemática viva también sea transferido junto con el cuerpo de conocimientos ya elaborado. Es decir, lo más probable (aunque hay notables excepciones) es que la transferencia y el implante sólo lo sean de un cuerpo petrificado de conocimientos (sin la vida inquisitiva que lo anima) destinado a repetirse a manera de dogma en sucesivos actos de enseñanza escolar. Cuando este es el caso, la investigación que se realiza en una unidad académica de este tipo resulta forzada, inauténtica y, claro está, desconectada del aparato docente ya establecido.

Por el contrario, la sistemología interpretativa surgió como una respuesta institucional ante una inquietud inquisitiva. Esa inquietud inquisitiva, esa necesidad e interés de enfrentar una problemática cognoscitiva, dio lugar a un cuerpo de conocimientos que siempre ha estado sometido a la dinámica propia de una práctica. Nuestro programa de postgrado fue creado para cultivar (desplegar en un discurso enseñable, enseñar, discutir, permitir que los estudiantes contribuyan con la investigación que da lugar a lo enseñado) lo que hemos ido aprendiendo a lo largo de estos 20 años de búsqueda inquisitiva. "Cultivar" no quiere aquí decir simplemente enseñar o trasmitir; quiere decir, fundamentalmente, crear las condiciones para una actividad en la que el proceso de investigación se enriquezca y se propague. En este sentido, por ejemplo, nuestra maestría esta estructurada de manera tal que, desde el primer semestre, los estudiantes participan en alguno de nuestros proyectos de investigación. Esto lo hacen en una asignatura tipo taller que ocupa la columna vertebral de nuestro pensum.

La sistemología interpretativa no se constituye como un mero cuerpo de conocimientos que pretenden neutralidad a-política. Por el contrario, nuestra disciplina siempre ha estado movida por un interés práctico (en el buen sentido del término): el de promover y afianzar la búsqueda holística de sentido de nuestro quehacer institucional.

El "interés práctico" (en el sentido kantiano y, luego, habermasiano del término) es ese que se plantea el problema de lo que debe hacerse dentro de un contexto moral. Es ese que cuestiona y discute fines institucionales y no simplemente los toma como dogmas. La mayor parte de lo que hoy se hace en las universidades está dominado por un interés instrumental, no-práctico, que se conforma con atender el problema de los medios para fines dados e incuestionados. Se trata de una instrumentalidad que apoya y afianza el orden y estructura de poder establecidos; de una disposición a-crítica del pensamiento. Este afán instrumental concibe la enseñanza como la preparación de las "piezas" humanas ("recursos humanos") requeridas por un aparato social predefinido e incuestionado.

Por el contrario, bajo el tipo de interés práctico que ha caracterizado la sistemología interpretativa, la enseñanza es el proceso de formación de seres humanos críticos en búsqueda del sentido holístico de sus prácticas sociales e instituciones. Esto no es otra cosa que investigadores en sistemología interpretativa. Y un investigador sólo se puede formar investigando; incorporándose a una práctica de investigación. 
Aparte de los interrogantes fundadores de la sistemología interpretativa y del interés general práctico que la ha movido, el interés político por constituirnos en ejemplo de una práctica universitaria (expuesto en la sección 3) ha sido un importante propulsor de nuestra actividad. Quisiera hacer notar la recursión implícita en lo que estoy diciendo:

Una de las primeras instituciones que estudiamos bajo la égida de nuestro método sistémico-interpretativo fue la universidad. Armamos una "plataforma sistémico-interpretativa" en términos de la cual discutir, por una parte, el papel actual de las universidades nacionales en Venezuela, y, por la otra, los posibles sentidos que, en general, pudiesen justificar la institución universitaria. Este trabajo nos develó una universidad deseable del tipo enunciado en la sección 3 y una universidad actual muy alejada de tales principios. Esa concepción de universidad deseable estaba en perfecta armonía con nuestra búsqueda de sentido holístico (nuestro interés práctico). De este modo surgió una íntima armonía entre nuestra actividad particular propia de la sistemología interpretativa y el ideal político de universidad que sosteníamos. En estos términos, nos resultó claro que el cuidadoso cultivo de una buena práctica de la sistemología interpretativa era el mejor modo de acción política universitaria: debíamos convertirnos en ejemplo institucional para reafirmar esa concepción de universidad; $y$, por ende, de búsqueda de sentido holístico. ¿Lo logramos? -se me preguntará. Sí, en cierta medida. No, como quisiéramos. Pero, no puede ser de otro modo. Una práctica no puede lograr a cabalidad el bien que persigue. Siempre tiene que ser posible -y necesario- hacerlo mejor. De lo contrario, el impulso vital de práctica se mengua. $Y$ con esto del "impulso vital de la práctica" cierro este circunloquio sobre el tema de la vinculación entre investigación y enseñanza de postgrado.

\section{Conclusión}

En la sección 5.1 comencé diciendo que hubo tres condiciones fundamentales que contribuyeron de manera decisiva en el modo de vinculación a priori que se observa entre investigación y enseñanza de postgrado en la práctica de la sistemología interpretativa. Lo que estas tres condiciones hicieron posible fue, precisamente, ese "impulso vital" necesario para que la investigación y la enseñanza de postgrado en una unidad académica se den a partir de ese vínculo a priori entre ambas; el cual, a su vez, encuentra la práctica como nicho socio-laboral. Con todo esto, espero haber ilustrado la tesis fundamental de este opúsculo; a saber:

1. La autenticidad y calidad de las actividades de investigación y enseñanza de postgrado en una universidad descansan, por lo menos en parte, en la posibilidad de la vinculación a priori entre ambas actividades.

2. Esa vinculación sólo es viable bajo la forma socio-laboral de una práctica (en el sentido antes explicado).

3. Esa práctica universitaria, en la cual la investigación y la enseñanza de postgrado son las dos caras de una misma moneda, requiere de un "impulso vital”. Tal impulso, en su más animada condición, resulta de una 
auténtica e íntima síntesis entre un interés inquisitivo y un interés prácticopolítico por el bien del mundo que habitamos.

\section{Bibliografía Citada}

Fuenmayor, R.L. (1991a), The Roots of Reductionism: A Counter-Ontoepistemology for a Systems Approach. Syst. Pract 4, 419-447.

Fuenmayor, R.L. (1991b), The Self-Referential Structure of an Everyday-Living Situation: A Phenomenological Ontology for Interpretive Systemology. Syst. Pract 4, 449-472.

Fuenmayor, R.L. (1991c), Truth and Openness: An Epistemology for Interpretive Systemology. Syst. Pract4, 473-490.

Fuenmayor, R.L. (2001a), Interpretando Organizaciones. Una Teoría Sistémico-Interpretativa de Organizaciones, Consejo de Publicaciones de la Universidad de Los Andes, Mérida-Venezuela.
Fuenmayor, R.L., (2001b), "Entre la mediocridad institucional y el dominio imperial de lo instrumental" en Dávila J. (compilador): Pensando en la Universidad, Editorial Panapo, Venezuela. pp. 67-88.

Heidegger, M. (1993), On the essence of truth. In Basic Writings. Routledge \& Kegan Paul. London, pp. 115-138.

Maclntyre, A. (1985), After Virtue: a study in moral theory. Duckworth, Great Britain.

Wittgenstein, L. (1978), Philosophical Investigations, Basil Blackwell, Oxford. 